\title{
Corneal Toxicity Induced by ABT-414 Therapy for Glioblastoma Multiforme: A Case Report
}

\author{
Hamoon Eshraghi ${ }^{a} \quad$ Dimosthenis Mantopoulos $^{a}$ Leejee H. Suh ${ }^{b}$ \\ Francisco Zaldana ${ }^{c}$ Howard F. Fine ${ }^{a}$ \\ aDepartment of Ophthalmology, Rutgers Robert Wood Johnson Medical School, New \\ Brunswick, NJ, USA; b Department of Ophthalmology, Columbia University College of \\ Physicians and Surgeons, New York, NY, USA; 'Department of Pathology, Rutgers Robert \\ Wood Johnson Medical School, New Brunswick, NJ, USA
}

\section{Keywords}

Corneal toxicity $\cdot$ ABT-414 - EGFR inhibitor

\begin{abstract}
Background: To describe a case of corneal toxicity associated with the use of the epidermal growth factor receptor (EGFR) inhibitor ABT-414 in the treatment of glioblastoma multiforme. Case Presentation: Case report of a 56-year-old male with glioblastoma multiforme who developed mild painless blurred vision after systemic treatment with the investigational EGFR inhibitor ABT-414. The patient had best corrected visual acuity of 20/60 right eye and 20/50 left eye. Ophthalmic examination revealed corneal toxicity with whorl-like opacities in the inferior interpalpebral cornea. The patient was treated with topical fluoromethalone and lifitegrast, and his ocular symptoms as well as the corneal findings improved. Conclusions: The systemic use of EGFR inhibitor ABT-414 may be associated with corneal toxicity and the effects are reversible with treatment.




\section{Background}

ABT-414 is an investigational epidermal growth factor receptor (EGFR) monoclonal immunoglobulin G1 (IgG1) antibody being investigated for use in a protocol with temozolamide and radiation in the treatment of glioblastoma multiforme (GBM), the most common and aggressive malignant primary brain tumor. As an antibody-drug conjugate, the drug works by not only targeting EGFR, but also by releasing a cytotoxic agent, monomethyl auristatin $\mathrm{F}$ [1]. ABT-414 is stable in the bloodstream and only releases its cytotoxic agent when inside targeted cancer cells. ABT-414 is also being investigated for use in the treatment of squamous cell cancers.

ABT-414 has been shown to have treatment adverse effects, and the most common of these is ocular toxicity. A recent study of 45 patients showed that 40 had some degree of ocular toxicity and 12 had grade III/IV ocular toxicity [2].

We report the corneal findings and treatment of a patient undergoing investigational therapy with ABT-414.

\section{Case Presentation}

A 56-year-old man with seizures and slurring of his speech was diagnosed with a frontoparietal GBM, pathologically confirmed following resection via right-sided craniotomy (Fig. 1). Analysis of the GBM was positive for EGFR amplification. He was treated with ABT-414, temozolomide, and radiation therapy in an investigational protocol. The protocol called for intravenous infusion of ABT-414 dosed at $175 \mathrm{mg}(2 \mathrm{mg} / \mathrm{kg}$ ) every 2 weeks.

Following the third cycle of ABT-414, the patient developed mild painless blurred vision in both eyes that interfered with his reading. He was referred to ophthalmology. There was no past ocular history. Ophthalmic examination disclosed best corrected visual acuity of 20/60 right eye and 20/50 left eye. Pupillary examination, motility, confrontation visual fields, and intraocular pressures were normal. Slit lamp examination of the lids, lashes, lacrimal glands and ducts, conjunctiva, and sclera was unremarkable. Corneal examination was notable for whorl-like opacities in the inferior interpalpebral cornea (Fig. 2). Dilated fundus examination was unremarkable. Specular microscopy disclosed normal endothelial cell morphology and cell density for age, 2,280 and 2,186 for the right and left eyes, respectively.

After discussion with the oncology team, the dosing of ABT 414 was decreased to $110 \mathrm{mg}$ $(1.25 \mathrm{mg} / \mathrm{kg})$. Treatment was initiated with fluoromethalone $0.1 \%$ and lifitegrast in both eyes one drop twice daily, and the patient noted a gradual improvement in symptoms over several weeks. Corneal findings also improved but did not entirely resolve.

\section{Discussion and Conclusions}

ABT-414 is administered via intravenous infusion into the systemic circulation. We hypothesize that, because the cornea is an avascular structure, the antibody-drug conjugate may be secreted in tears and delivered to the cornea. ABT-414 is an IgG1 antibody, and IgG antibodies are known to be secreted in tears in small quantities [3].

Additionally, it is possible that the drug is delivered systemically to the limbal stem cells, which possess a blood supply. The corneal basal epithelial cells express EGFR. Basal cells at the limbus have been shown to have 4.5 times greater EGFR signaling than the basal cells in 
the central cornea [4]. EGF is an important polypeptide growth factor produced in the lacrimal gland that is a potent stimulator of cell growth and differentiation. Inhibition of EGFR in the basal epithelial cells by ABT-414 could lead to degeneration of the rapidly dividing basal epithelial cells, leading to corneal epitheliopathy.

The pathophysiology of this keratopathy may be similar to that of cornea verticillata, which also commonly manifests as whorl-like patterns in the inferior interpalpebral cornea. Cornea verticillata, also known as vortex keratopathy, is associated with Fabry disease as well as with toxicity from certain drugs, most commonly amiodarone. The exact pathogenesis of the condition is poorly understood, but in both Fabry disease and drug-induced verticillata, intralysosomal inclusion bodies have been observed. The condition has also been associated with use of another anti-EGFR agent, vandetanib [5, 6]. In vandetanib-associated corneal toxicity, while the corneal findings did not resolve, the associated ophthalmic symptoms did improve after a decrease in the dosage. Other reports of drug-induced verticillata have also been found to be reversible [7].

In our patient, the corneal findings were significant because the patient did experience painless blurring of his vision that interfered with his activities of daily living. With treatment, his symptoms did improve although the corneal epithelial changes did not resolve entirely. In the future, it is important to raise awareness of the possibility of ocular symptoms in those receiving treatment with ABT-414 or other EGFR inhibitors so that patients could receive treatment to lessen their symptoms.

\section{Statement of Ethics}

Patient's next of kin has granted consent for publication.

\section{Disclosure Statement}

The authors have no conflicts of interest.

\section{Author Contributions}

Dr. Eshraghi and Dr. Mantopoulos wrote and reviewed the manuscript; Dr. Mantopoulos examined the patient; Dr. Fine and Dr. Suh interpreted and commented on the data and reviewed the manuscript; and Dr. Zaldana reviewed and commented on the pathology of the patient. All authors read, edited, and approved the case report.

\section{References}

1 Phillips AC, Boghaert ER, Vaidya KS, Mitten MJ, Norvell S, Falls HD, et al. ABT-414, an Antibody-Drug Conjugate Targeting a Tumor-Selective EGFR Epitope. Mol Cancer Ther. 2016 Apr;15(4):661-9.

2 Reardon DA, Lassman AB, van den Bent M, Kumthekar P, Merrell R, Scott AM, et al. Efficacy and safety results of ABT-414 in combination with radiation and temozolomide in newly diagnosed glioblastoma. Neuro Oncol. 2017 Jul;19(7):965-975.

3 Agarwal S, Agarwal A, Apple DJ. Textbook of Ophthalmology. New Delhi: Jaypee Brothers Publishers; 2002.

4 Zieske JD, Wasson M. Regional variation in distribution of EGF receptor in developing and adult corneal epithelium. J Cell Sci. 1993 Sep;106(Pt 1):145-52. 
5 Yeh S, Fine HA, Smith JA. Corneal verticillata after dual anti-epidermal growth factor receptor and antivascular endothelial growth factor receptor 2 therapy (vandetanib) for anaplastic astrocytoma. Cornea. 2009 Jul;28(6):699-702.

6 Ahn J, Wee WR, Lee JH, Hyon JY. Vortex keratopathy in a patient receiving vandetanib for non-small cell lung cancer. Korean J Ophthalmol. 2011 Oct;25(5):355-7.

7 Hollander DA, Aldave AJ. Drug-induced corneal complications. Curr Opin Ophthalmol. 2004 Dec;15(6):5418.

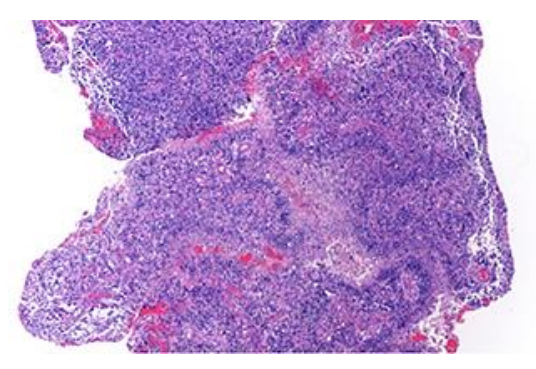

Fig. 1. Hematoxylin and eosin stain at $\times 40$ magnification diagnostic of glioblastoma multiforme with highgrade astrocytoma with nuclear atypia, cellular pleomorphism, mitotic activity, and thickened vascular walls. 


\section{Case Reports in Ophthalmology}
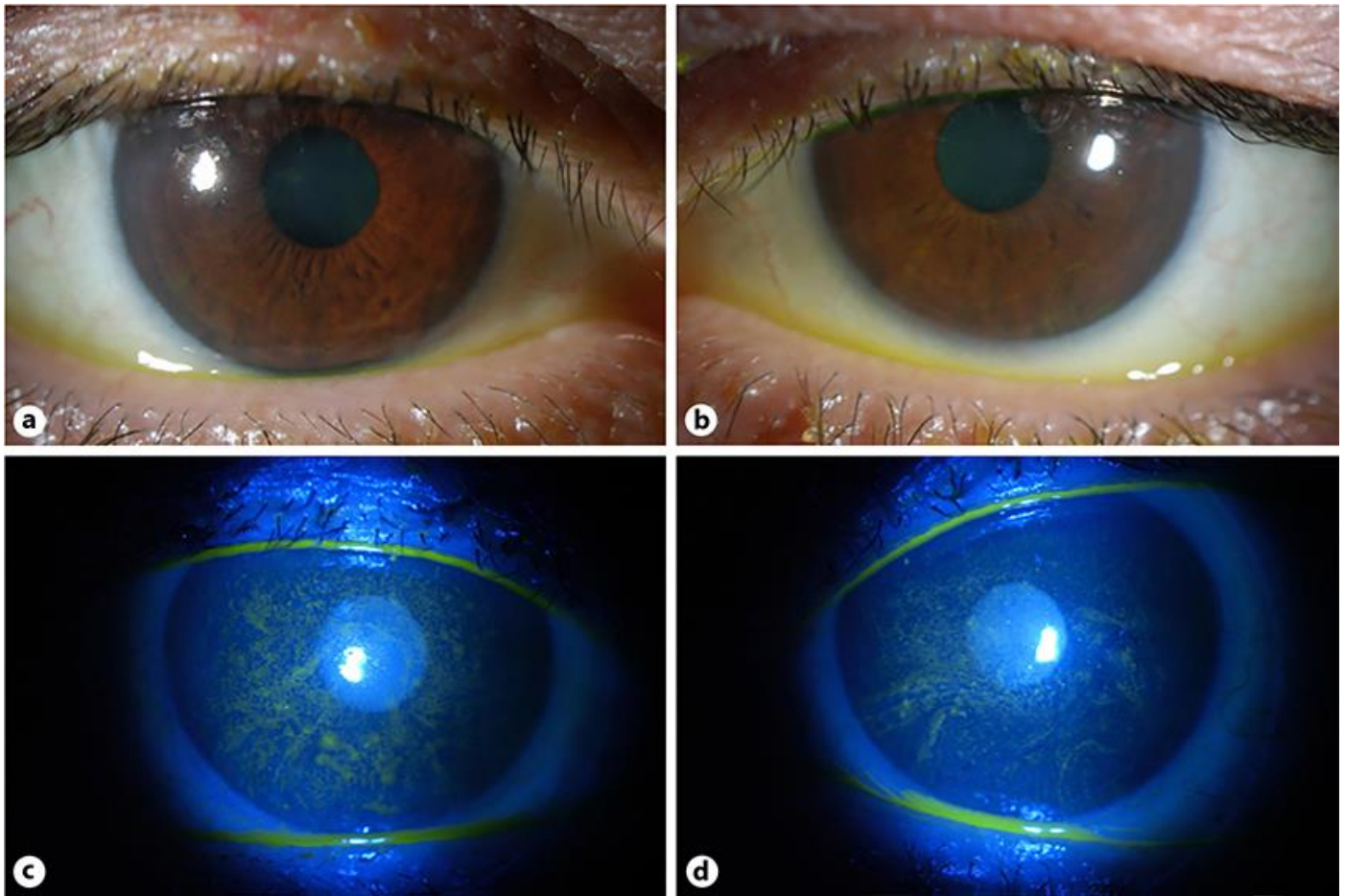

Fig. 2. a Color photograph of the right cornea demonstrating whorl-like subepithelial opacities in the inferior interpalpebral zone. $\mathbf{b}$ Color photograph of the left cornea showing similar findings as the left cornea. c Color photograph of right cornea with fluorescein dye demonstrating superficial punctate keratitis. d Color photograph of the left cornea with fluorescein dye showing similar findings as the right cornea. 